05

\title{
Особенности фокусировки спиновых волн в ферромагнетиках
}

\author{
(C) С.М. Бахарев ${ }^{1}$, С.П. Савченко ${ }^{1}$, А.П. Танкеев ${ }^{1,2}$ \\ ${ }^{1}$ Институт фризики металлов им. М.Н. Михеева УрО РАН, \\ Екатеринбург, Россия \\ ${ }^{2}$ Уральский федеральный университет им. Б.Н. Ельцина, \\ Екатеринбург, Россия \\ E-mail: bakharevsm@imp.uran.ru
}

(Поступила в Редакцию 22 марта 2018 г.)

Исследованы особенности фокусировки спиновых волн в ферромагнетиках с обменным взаимодействием магнитного момента с ближайшими и следующими за ними соседями. Показано, что в длинноволновом приближении фокусировка спиновых волн отсутствует: она наблюдается лишь для волновых векторов во второй половине зоны Бриллюэна (при $a q \geq \pi / 2$, где $a-$ постоянная решетки, $q-$ величина волнового вектора). Кроме того показано, что магноны в такого рода системе фокусируются вдоль направлений [110], а в направлениях [100] - дефокусируются. Обнаружено, что внешнее магнитное поле и поле магнитной анизотропии не приводят к изменению направлений фокусировки магнонов. Для отрицательного параметра обменного взаимодействия со вторыми соседями вблизи направлений [110] и [111] имеет место каустика магнонов, вблизи которой резко возрастает интенсивность спин-волнового поля.

Работа выполнена в рамках государственного задания по теме „Спин“ ААААA18-118020290104-2 и проекта № 32-1.1.3.5 Программы фундаментальных исследований Президиума РАН согласно контракту Минобрнауки № 14.Z50.31.0025, а также гранта РФФИ мол_а (проект № 18-32-00139).

DOI: 10.21883/FTT.2018.12.46725.077

\section{1. Введение}

Настоящая работа посвящена теоретическому исследованию фокусировки спиновых волн в одноосных ферромагнетиках. В системах с зависимостью частоты собственных колебаний от направления волнового вектора групповая и фазовая скорости не коллинеарны, что приводит к появлению преимущественных направлений в кристалле, вдоль которых происходит распространение изучаемых волн (или фокусировка).

Впервые фокусировка и связанные с ней эффекты исследовались на фононах при распространении тепловых импульсов в упруго анизотропных кристаллах [1-6]. В этих исследованиях отмечалась резкая анизотропия пространственного распределения потока энергии акустических колебаний различных поляризаций, которая напрямую связывалась с явлением фокусировки фононов.

Интерес к исследованию фокусировки магнонов появился позднее - в начале XXI века. Так, в работах $[7,8]$ методом бриллюэновского рассеяния света впервые обнаружена самофокусировка спиновых волн в гранатовых пленках [7] и пермаллоевых микрополосках [8]. Она проявлялась как пик интенсивности спиновых волн в конкретной точке на плоскости образца. Самофокусировка наблюдалась в „нелинейном режиме“ (с мощностью микроволнового излучателя 600-700 mW), однако в „линейном режиме“ (с мощностью излучателя $10 \mathrm{~mW}$ ) самофокусировка отсутствовала или проявлялась довольно слабо [7].

В работе [9] впервые была теоретически изучена фокусировка объемных и поверхностных магнитостати- ческих волн в тонких ферромагнитных пленках железоиттриевого граната $\mathrm{Y}_{3} \mathrm{Fe}_{5} \mathrm{O}_{12}$ (ЖИГ) и $\mathrm{Fe}$ во внешнем магнитном поле, а в работе [10] (тем же составом авторов) - в антиферромагнитных пленках $\mathrm{MnF}_{2}, \mathrm{FeF}_{2}$ и $\mathrm{GdAlO}_{3}$. Для получения картин фокусировки авторы численно решили магнитостатические уравнения Максвелла совместно с уравнениями движения для намагниченности в пределе длинных волн. В работе [9] ими были учтены как дипольное, так и обменное взаимодействия. Им удалось показать, что для объемных образцов учет обменного взаимодействия качественно не меняет картину фокусировки, однако для тонких пленок его учет кардинально меняет фокусировку спиновых волн. Показано также, что магнитостатические поверхностные волны фокусируются хуже, чем объемные волны. В обоих случаях показано, что направления фокусировки управляются частотой и приложенным магнитным полем.

В работе [11] были рассчитаны картины фокусировки магнонов в образцах ЖИГ для двумерной решетки. Было показано, что фокусировка спиновых волн в коротковолновом диапазоне возможна без учета магнитной анизотропии, дипольных эффектов и внешнего магнитного поля. Фокусировка магнонов имела место для волновых векторов во второй половине зоны Бриллюэна, однако для длинных волн она отсутствовала. Было показано, что картина фокусировки и формирование соответствующих каустик зависят от знака константы обменного взаимодействия, отвечающей за взаимодействие магнитного момента со вторыми соседями. В работе [11] авторы показали, что изменение величины внешнего магнитного поля приводит к вращению поверхности постоянной 
энергии спиновых волн и к изменению направлений фокусировки магнонов. Последний вывод нуждается в проверке.

Проведенный нами детальный анализ результатов работы [11] показал, что авторы допустили ряд неточностей и, по всей видимости, вывод о том, что внешним магнитным полем можно управлять фокусировкой магнонов, является ошибочным. Мы показали, что ни внешнее магнитное поле, ни поле магнитной анизотропии не приводят к изменению направлений фокусировки спиновых волн. Последнее обусловлено только обменным взаимодействием магнитных моментов атомов.

Помимо анизотропной дисперсии магнонов наблюдение магнитной фокусировки обычно требует создания спиновых волн в локализованной области. Этот эффект экспериментально можно наблюдать при более низких частотах (порядка 3-10 GHz) с помощью локализованных микроволновых токов, которые возбуждают переменные магнитные поля. В качестве точечного источника магнонов также могут служить магнитные антиточки, которые формируют внутреннее неоднородное магнитное поле $[12,13]$.

Цель настоящей работы - исследование особенностей фокусировки спиновых волн в одноосных ферромагнетиках. Для ее достижения мы рассмотрели ферромагнетик, полная энергия которого складывается из обменной энергии, зеемановской энергии взаимодействия магнитного момента с магнитным полем и энергии магнитной анизотропии [14].

Используя феноменологический подход, в котором динамика магнитного момента описывается с помощью уравнений движения без затухания при условии сохранения величины магнитного момента, мы рассчитали спектр магнонов в трехмерной простой кубической решетке для двух случаев:

1) с учетом обменного взаимодействия только с ближайшими соседями;

2) с учетом обменного взаимодействия с ближайшими и следующими за ними соседями.

Поскольку групповая скорость перпендикулярна изоэнергетической поверхности, зная спектр спиновых волн, можно определить направлении фокусировки и дефокусировки магнонов. Для количественного описания явления фокусировки будут использованы два подхода, разработанные для фононов: 1) метод, основанный на вычислении коэффициента усиления и 2) метод, основанный на вычислении углового распределения плотности состояний.

Первый метод был разработан Марисом [4] в 1971 г. и развит в работах $[3,5,15-18]$ для количественной оценки фокусировки фононов в кубических кристаллах. Коэффициент усиления потока фононов определяется как отношение потока тепла данной поляризации в исследуемом кристалле к соответствующему потоку тепла в изотропной среде для выбранного направления волнового вектора. Этот коэффициент показывает во сколько раз изменится интенсивность потока данной колебательной моды по сравнению с изотропной средой. Коэффициент усиления позволяет рассчитать положения параболических линий и каустик. На этих линиях кривизна изоэнергетической поверхности обращается в нуль, и, поскольку коэффициент усиления обратно пропорционален кривизне, он имеет сингулярность. Последнее обстоятельство указывает на каустику.

Второй метод разработан в работах $[19,20]$ для вычисления угловых распределений плотности фононных состояний (ПФС) квазипоперечных фононных мод. Он основан на анализе структуры поверхности постоянной энергии поперечных мод при наличии вогнутых участков на этой поверхности и расчете углов, характеризующих фокусировку фононов. Плотность состояний определяется отношением сектора углов групповых скоростей фононов, распространяющихся в изотропной среде, к сектору углов групповых скоростей фононов, распространяющихся в рассматриваемом кристалле. В области фокусировки ПФС больше единицы, в области дефокусировки - меньше единицы. Этот подход предполагается использовать для количественной оценки плотности состояний магнонов при наличии вогнутых областей на изоэнергетической поверхности.

Как уже отмечалось выше, в настоящей работе рассматриваются только магнитноодноосные кристаллы. К ним можно отнести, например, кубические ферритыгранаты состава $R_{3} \mathrm{Fe}_{5} \mathrm{O}_{12}$, где символ $R$ означает элемент Y или редкоземельные металлы $\mathrm{Sm}, \mathrm{Eu}, \mathrm{Ho}$ В этих материалах можно создать одноосную магнитную анизотропию, подвергая их, например, отжигу в магнитном поле [21,22]. Заметим, что наведенная при этом анизотропия должна „подавлять“ магнитостатические эффекты, как это имеет место в случае магнитно-одноосных ферритов-гранатов, используемых при создании устройств с цилиндрическими магнитными доменами (ЦМД): важнейшей характеристикой ЦМД-пленки является так называемый фактор качества $Q=H_{a} /\left(4 \pi M_{0}\right)>1\left(H_{a}-\right.$ поле анизотропии, $M_{0}-$ намагниченность насыщения) [23]. Поэтому следует ожидать, что в указанных материалах ввиду малости размагничивающих полей фокусировка обменных спиновых волн будет более эффективной, нежели фокусировка магнитостатических типов возбуждений.

Показано, что в длинноволновом приближении спектр магнонов изотропен - он не зависит от направления волнового вектора, поэтому в этом случае фокусировка спиновых волн отсутствует. Зависимость частоты магнонов от направления волнового вектора возникает во второй половине зоны Бриллюэна в области терагерцовых частот.

Анализ структуры изоэнергетических поверхностей показал, что в первом случае (в модели, учитывающей обменное взаимодействие только с ближайшими соседями) в плоскости грани куба $\{100\}$ фокусировка магнонов происходит в направлении [110] в области частот $v \geq 3.11 v_{E}\left(v_{E}-\right.$ параметр обменного взаимодействия). При $v=4.06 v_{E}$ коэффициент усиления в 
направлении фокусировки $A_{[110]}=47$. В диагональной плоскости $\{110\}$ фокусировка магнонов происходит в том же диапазоне частот в направлении [111]. При $v=4.06 v_{E}$ коэффициент усиления $A_{[111]}=5$.

Во втором случае, с параметром обменного взаимодействия со вторыми соседями $\xi=0.2$, спектр спиновых волн имеет слабую анизотропию и, соотвественно, слабую фокусировку. В направлении фокусировки [110] коэффициент усиления $A_{[110]}=3.5$. Однако в случае $\xi=-0.2$ форма изоэнергетических поверхностей при частотах $v \geq 0.53 v_{E}$ принимает более сложный вид: в окрестности направлений [110] и [111] на них появляются вогнутые участки, что приводит к расходимости коэффициента усиления и появлению каустики магнонов вблизи этих направлений.

Произведена оценка плотности состояний магнонов для параметра $\xi=-0.2$ в плоскости грани куба: при частоте $v=0.83 v_{E}$. В области фокусировки [110] плотность состояний больше в 175 раз, чем в области дефокусировки магнонов [100].

На примере магнетика ЖИГ оценили частоты, при которых имеет место фокусировка магнонов и формирование каустик: $v=0.048-1.3 \mathrm{THz}$.

\section{2. Спектр спиновых волн в одноосных ферромагнетиках}

Плотность энергии магнитно-одноосного ферромагнетика с точностью до членов, квадратичных по намагниченности $\mathbf{M}(\mathbf{r})$, записывается в виде [14,24]

$$
\begin{aligned}
W(\mathbf{r})= & -K_{a}\left(\frac{M_{z}(\mathbf{r})}{M_{0}}\right)^{2}-\mathbf{M}(\mathbf{r}) \mathbf{H} \\
& -\frac{1}{M_{0}^{2}} \sum_{\boldsymbol{\delta} \neq 0} J(\boldsymbol{\delta}) \mathbf{M}(\mathbf{r}) \mathbf{M}(\mathbf{r}+\boldsymbol{\delta}),
\end{aligned}
$$

где $M_{0}$ - намагниченность насыщения, $\mathbf{r}-$ положение атома в кристалле, $K_{a}$ - константа анизотропии, $J$ - константа обменного взаимодействия. Здесь первое слагаемое соответствует энергии кристаллографической анизотропии, второе - зеемановской энергии магнетика во внешнем магнитном поле Н. Последнее слагаемое соответствует обменной энергии. Суммирование ведется по атомам с координатами $\boldsymbol{\delta}$.

Для получения спектра спиновых волн используем классический подход, основанный на решении линейных уравнений движения для намагниченности $\mathbf{M}(\mathbf{r})$ $\left(\mathbf{M}=\mathbf{M}_{0}+\Delta \mathbf{M},|\Delta \mathbf{M}| \ll\left|\mathbf{M}_{0}\right|\right)$

$$
\dot{\mathbf{M}}=-\gamma\left[\mathbf{M} \times \mathbf{H}^{\mathrm{eff}}\right],
$$

где $\gamma$ - фактор спектроскопического расщепления (фактор Ланде), $\gamma>0 ; \mathbf{H}^{\mathrm{eff}}-$ эффективное магнитное поле, действующее на намагниченность и определяемое соотношением [14]

$$
\mathbf{H}^{\mathrm{eff}}=-\frac{\delta W}{\delta \mathbf{M}}
$$

Поскольку в ферритах-гранатах затухание колебаний магнитного момента чрезвычайно мало [22], то в уравнении движения (2) мы им пренебрегли. Феноменологически затухание можно учесть, следуя уравнению ЛандауЛифшица, либо Гильберта [24,25].

Перейдем от системы координат $X Y Z$, связанной с осями кристалла, в систему координат $X^{\prime} Y^{\prime} Z^{\prime}$, в которой вектор $\mathbf{M}_{0}$ направлен вдоль оси $O Z^{\prime}$. В случае одноосного кристалла можно, без потери общности, считать, что поле Н лежит в одной плоскости с осями $O Z$ и $O Z^{\prime}$. Тогда компоненты намагниченности в локальной системе координат параметризуются в виде

$$
\begin{gathered}
M_{x}=M_{x} \cos \Theta+M_{z} \sin \Theta, \\
M_{y}=M_{y}, \\
M_{z}=-M_{x} \sin \Theta+M_{z} \cos \Theta,
\end{gathered}
$$

где $\Theta$ - угол между осями $O Z$ и $O Z^{\prime}$. Направим поле Н вдоль оси $O Z$ и запишем плотность энергии (1) в компонентах $M_{\hat{x}}, M_{\dot{y}}$ и $M_{\dot{z}}$

$$
\begin{aligned}
& W(\mathbf{r})=-\frac{K_{a}}{M_{0}^{2}}\left(M_{\grave{x}}^{2} \sin ^{2} \Theta+M_{\grave{z}}^{2} \cos ^{2} \Theta-2 M_{\hat{x}} M_{\dot{z}} \sin \Theta \cos \Theta\right) \\
& -\left(-M_{\hat{x}} \sin \Theta+M_{\bar{z}} \cos \Theta\right) H-\frac{1}{M_{0}^{2}} \sum_{\delta \neq 0} J(\boldsymbol{\delta}) \mathbf{M}(\mathbf{r}) \mathbf{M}(\mathbf{r}+\boldsymbol{\delta}) .
\end{aligned}
$$

Ищем минимум энергии основного состояния $W_{0}$ по углу $\Theta$

$$
\frac{\partial W_{0}}{\partial \Theta}=2 K_{a} \sin \Theta \cos \Theta+M_{0} H \sin \Theta=0 .
$$

Из равенства (8) следует, что энергия основного состояния $W_{0}$ имеет минимум в двух случаях:

$$
\sin \Theta=0, \quad \cos \Theta=-\frac{H}{H_{0}},
$$

где $H_{a}=2 K_{a} / M_{0}$ - поле анизотропии. Первое из этих решений соответствует минимуму энергии для любой величины поля $H$ при положительной константе анизотропии $K_{a}>0$ (когда ось симметрии $O Z$ является осью легкого намагничивания), а также при $K_{a}<0$ (когда ось симметрии $O Z$ является осью трудного намагничивания) для полей $H>|H|$. Второе решение реализуется при $K_{a}<0$ для $H<\left|H_{a}\right|$ : с увеличением $H$ магнитный момент постепенно поворачивается от базисной плоскости по направлению к оси $O Z$ и при $H=\left|H_{a}\right|$ становится параллельным последней - наступает насыщение [14].

В линейном по компонентам намагниченности приближении из выражения (2) с учетом (3) и (8) получаем

$$
\begin{aligned}
\dot{M}_{\hat{x}}(\mathbf{r})= & -\gamma \frac{M_{\hat{y}}(\mathbf{r})}{M_{0}}\left(2 K \cos ^{2} \Theta+H M_{0} \cos \Theta+\sum_{\boldsymbol{\delta} \neq 0} J(\boldsymbol{\delta})\right) \\
& +\frac{\gamma}{M_{0}} \sum_{\boldsymbol{\delta} \neq 0} J(\boldsymbol{\delta}) M_{\hat{y}}(\mathbf{r}+\boldsymbol{\delta}),
\end{aligned}
$$




$$
\begin{gathered}
\dot{M}_{\hat{y}}(\mathbf{r})=\gamma \frac{M_{\dot{x}}(\mathbf{r})}{M_{0}}\left(2 K \cos (2 \Theta)+H M_{0} \cos \Theta-\sum_{\delta \neq 0} J(\boldsymbol{\delta})\right) \\
+\frac{\gamma}{M_{0}} \sum_{\delta \neq 0} J(\boldsymbol{\delta}) M_{\dot{x}}(\mathbf{r}+\boldsymbol{\delta}), \\
\dot{M}_{\bar{z}}(\mathbf{r}) \approx 0 .
\end{gathered}
$$

Поскольку величина магнитного момента со временем не меняется $(\dot{M}(\mathbf{r})=0)$, то для компонент намагниченности должно выполняться следующее равенство: $\dot{M}_{\hat{x}}(\mathbf{r}) M_{\hat{x}}(\mathbf{r})+\dot{M}_{\hat{y}}(\mathbf{r}) M_{\hat{x}}(\mathbf{r})=0$ Поэтому решение системы уравнений (10), (11) ищем в виде плоских волн:

$$
\mathbf{M}=\mathbf{M}_{0} e^{(\omega t-\mathbf{q r})} .
$$

В результате получаем спектр спиновых волн в одноосных ферромагнетиках во внешнем магнитном поле $H$ в виде

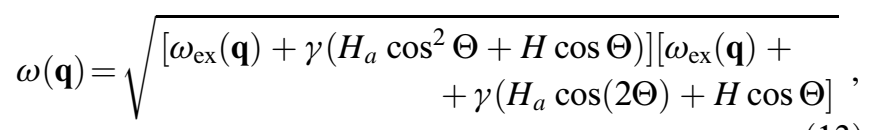

где

$$
\omega_{\mathrm{ex}}(\mathbf{q})=\frac{\gamma}{M_{0}} \sum_{\boldsymbol{\delta} \neq 0} J(\boldsymbol{\delta})\left(1-e^{-i \mathbf{q} \boldsymbol{\delta}}\right)
$$

- частота спиновых волн, связанная с обменным взаимодействием. Аналогичный результат получается методом вторичного квантования [24].

При $\sin \Theta=0$ спектр (13) приводится к виду

$$
\omega(\mathbf{q})=\omega_{\mathrm{ex}}(\mathbf{q})+\gamma\left(H+H_{a}\right) .
$$

При $\cos \Theta=-H / H_{a}$ при $H<\left|H_{a}\right|, H_{a}<0$ для поля $H$, направленного вдоль оси $O Z$, спектр (13) приводится к виду

$$
\omega(\mathbf{q})=\sqrt{\omega_{\mathrm{ex}}(\mathbf{q})\left[\omega_{\mathrm{ex}}(\mathbf{q})+\gamma\left|H_{a}\right|\left(1-H / H_{a}\right)^{2}\right]} .
$$

В рассмотренных случаях поле анизотропии и внешнее магнитное поле приводят к появлению щели в спектре магнонов, но не изменяют его угловые зависимости и, соотвественно, направления фокусировки спиновых волн. Поэтому вывод работы [11] об изменении направлений фокусировки магнонов во внешнем магнитном поле является ошибочным. При анализе фокусировки спиновых волн, как было отмечено выше, нас будет интересовать область терагерцовых частот. В этом случае $\omega_{\mathrm{ex}}(\mathbf{q}) \gg \gamma\left|H_{a}\right|$, а учет постоянного магнитного поля $H$ не влияет на фокусировку магнонов. Поэтому в рассматриваемой задаче достаточно учесть обменное взаимодействие спинов электронной системы и считать, что

$$
\omega(\mathbf{q})=\omega_{\mathrm{ex}}(\mathbf{q}),
$$

где $\omega_{\text {ex }}(\mathbf{q})$ определяется соотношением (14). С учетом взаимодействия с двумя ближайшими соседями в простой кубической решетке $\omega_{\mathrm{ex}}(\mathbf{q})$ можно представить в виде

$$
\begin{aligned}
\omega_{\mathrm{ex}}(\mathbf{q})= & 2 \omega_{E}\left[3-\cos \left(q_{x} a\right)-\cos \left(q_{y} a\right)-\cos \left(q_{z} a\right)\right. \\
& +2 \xi\left(3-\cos \left(q_{x} a\right) \cos \left(q_{z} a\right)-\cos \left(q_{y} a\right) \cos \left(q_{z} a\right)\right. \\
& \left.\left.-\cos \left(q_{x} a\right) \cos \left(q_{y} a\right)\right)\right],
\end{aligned}
$$

где $\omega_{E}=\gamma J_{1} / M_{0}\left(J_{1}-\right.$ постоянная обменного взаимодействия для ближайших соседей $), \xi=J_{2} / J_{1}\left(J_{2}-\right.$ постоянная обменного взаимодействия для соседей, следующих за ближайшими), $a$ - параметр решетки. В длинноволновом приближении $(a q \ll 1)$ выражение $(18)$ преобразуется к виду

$$
\omega_{\mathrm{ex}}(q)=\omega_{E}(1+4 \xi)(a q)^{2} .
$$

Из формулы (19) следует, что есть ограничение на выбор параметра обменного взаимодействия со вторыми соседями: $|\xi|<0.25$. В противном случае необходимо учитывать взаимодействие с тремя и более соседями. Отметим, что спектр в виде (18) позволяет проводить анализ фокусировки спиновых волн без привязки к конкретному кристаллу. Для этого достаточно задать отношения частот $\omega / \omega_{E}$ и безразмерный волновой вектор $a q$ (где $\omega_{E}$ - параметр обменного взаимодействия).

Групповая скорость магнонов определяется следующим соотношением:

$$
\mathbf{V}(\mathbf{q})=\frac{\partial \omega(\mathbf{q})}{\partial \mathbf{q}}=V_{n} \mathbf{n}+V_{\theta} \mathbf{e}_{\theta}+V_{\varphi} \mathbf{e}_{\varphi},
$$

где

$$
V_{n}=\frac{\partial \omega}{\partial q}, V_{\theta}=\frac{1}{q} \frac{\partial \omega}{\partial \theta}, V_{\varphi}=\frac{1}{q \sin \theta} \frac{\partial \omega}{\partial \varphi},
$$

а векторы

$$
\begin{aligned}
& \mathbf{n}=\mathbf{q} / q=(\sin \theta \cos \varphi, \sin \theta \sin \varphi, \cos \theta), \\
& \mathbf{e}_{\theta}=(\cos \theta \cos \varphi, \cos \theta \sin \varphi,-\sin \theta), \\
& \mathbf{e}_{\varphi}=(-\sin \varphi, \cos \varphi, 0)
\end{aligned}
$$

образуют тройку взаимно ортогональных векторов. Углы $\theta$ и $\varphi$ задают направление волнового вектора $\mathbf{q}$ (в системе координат по ребрам куба угол $\theta$ отсчитывается от оси $Z$, а угол $\varphi-$ от оси $X)$. Компоненты групповой скорости $V_{n}, V_{\theta}$ и $V_{\varphi}$ для спектра (18) можно представить в виде скалярных произведений

$$
\begin{gathered}
V_{n}(\mathbf{q})=\left(\mathbf{B}_{1}+\mathbf{B}_{2}\right) \mathbf{n}, \quad V_{\theta}(\mathbf{q})=\left(\mathbf{B}_{1}+\mathbf{B}_{2}\right) \mathbf{e}_{\theta}, \\
V_{\varphi}=\left(\mathbf{B}_{1}+\mathbf{B}_{2}\right) \mathbf{e}_{\varphi},
\end{gathered}
$$

где функции-векторы $\mathbf{B}_{1}(\mathbf{q})$ и $\mathbf{B}_{2}(\mathbf{q})$ определяются следующим образом

$$
\begin{gathered}
\mathbf{B}_{1}(\mathbf{q})=2 a \omega_{E}\left(\sin \left(q_{x} a\right), \sin \left(q_{y} a\right), \sin \left(q_{z} a\right)\right), \\
\mathbf{B}_{2}(\mathbf{q})=2 \xi\left(B_{1 x}\left[\cos \left(q_{y} a\right)+\cos \left(q_{z} a\right)\right],\right. \\
\left.B_{1 y}\left[\cos \left(q_{x} a\right)+\cos \left(q_{z} a\right)\right], B_{1 z}\left[\cos \left(q_{x} a\right)+\cos \left(q_{y} a\right)\right]\right) .
\end{gathered}
$$


В симметричных направлениях [100], [110] и [111] угловые компоненты групповой скорости $V_{\theta}=V_{\varphi}=0$. Поэтому в этих направлениях групповая и фазовая скорости коллинеарны. Отметим, что в плоскости грани куба и диагональной плоскости угловая компонента $V_{\varphi}=0$ для любого угла $\theta$.

\section{3. Коэффициент усиления потока магнонов}

Для количественного описания эффекта фокусировки магнонов применим метод, развитый Марисом в работе [4] для оценки фокусировки фононов. Он воспользовался моделью изотропной среды как системой сравнения и ввел понятие коэффициента усиления потока фононов $A(\theta, \varphi)$ 一 „enhancement factor", который также известен [1], как ,amplification factor“. Согласно [4] коэффициент $A(\theta, \varphi)$ определяется как отношение потока фононов данной поляризации для выбранного направления волнового вектора к соответствующему потоку в изотропной среде. Позже в работе [15] было показано, что коэффициент усиления характеризует изоэнергетическую поверхность и обратно пропорционален ее гауссовой кривизне, $A \sim 1 / K$.

По аналогии с работой $[15]$ коэффициент усиления $A(\mathbf{q})$, характеризующий различие потоков магнонов в анизотропном случае $(a q \gtrsim \pi / 2)$ и изотропном случае $(a q \ll 1)$, может быть определен через гауссову кривизну $K(\mathbf{q})$ следующим образом:

$$
A(\mathbf{q})=\frac{V_{n}}{V q^{2}}|K| .
$$

Следует отметить, что для длинноволновых фононов коэффициент усиления зависит только от направления волнового вектора (углов $\theta$ и $\varphi$ ), поскольку их спектр в длинноволновом приближении пропорционален модулю волнового вектора $(\omega \sim q)$. Однако для магнонов зависимость $\omega(q)$ нелинейная (см. выражение (18)), поэтому коэффициент усиления спиновых волн зависит не только от углов $\theta$ и $\varphi$, но и от модуля волнового вектора $(A=A(q, \theta, \varphi))$.

Согласно [26], при заданной частоте спиновых волн $\omega$ полная (или гауссова) кривизна поверхности $\mathbf{q}(\theta, \varphi)$ определяется следующим выражением:

$$
K(\mathbf{q})=\frac{L N-P^{2}}{E G-F^{2}},
$$

где коэффициенты $E, F$ и $G$ характеризуют первую квадратичную форму $d \mathbf{q}^{2}$, а коэффициенты $L, N$ и $P-$ вторую квадратичную форму $\left(-d \mathbf{q} / d \mathbf{n}_{V}\right)$, где $\mathbf{n}_{V}=\mathbf{V} / V-$ единичный вектор нормали к поверхности $\mathbf{q}(\theta, \varphi)$ (единичный вектор групповой скорости). Эти коэффициенты выражаются через частные производные волнового вектора $\mathbf{q}(\theta, \varphi)$ по углам $\theta$ и $\varphi[26]: E=\mathbf{q}_{\theta}^{2}, F=\mathbf{q}_{\theta} \mathbf{q}_{\varphi}$, $G=\mathbf{q}_{\varphi}^{2} ; L=q_{\theta \theta} \mathbf{n}_{V}, P=\mathbf{q}_{\theta \varphi} \mathbf{n}_{V}, N=\mathbf{q}_{\varphi \varphi} \mathbf{n}_{V}$. После вычисления частных производных волнового вектора $\mathbf{q}(\theta, \varphi)$ по углам $\theta$ и $\varphi$ для знаменателя в выражении (26) получаем

$$
E G-F^{2}=q^{4} \sin ^{2} \theta\left(\frac{V}{V_{n}}\right)^{2},
$$

а для коэффициентов $L, P$ и $N$ имеем следующие соотношения:

$$
\begin{gathered}
L=\frac{q}{V V_{n}}\left[-q \frac{V_{\theta}^{2}}{V_{n}} \frac{\partial V_{n}}{\partial q}+2 V_{\theta}\left(\frac{\partial V_{n}}{\partial \theta}-V_{\theta}\right)-V_{n}\left(\frac{\partial V_{\theta}}{\partial \theta}+V_{n}\right)\right] \\
P=\frac{q}{V V_{n}}\left[-q \sin \theta \frac{V_{\theta} V_{\varphi}}{V_{n}} \frac{\partial V_{n}}{\partial q}+V_{\theta} \frac{\partial V_{n}}{\partial \varphi}-V_{n} \frac{\partial V_{\theta}}{\partial \varphi}\right. \\
\left.+V_{\varphi}\left(\frac{\partial V_{n}}{\partial \theta}-2 V_{\theta} \sin \theta+V_{n} \cos \theta\right)\right], \\
N=\frac{q}{V V_{n}} \sin \theta\left[-q \frac{V_{\varphi}^{2}}{V_{n}} \sin \theta \frac{\partial V_{n}}{\partial q}-V_{n}\left(\frac{\partial V_{\varphi}}{\partial \varphi}+V_{n} \sin \theta\right.\right. \\
\left.\left.+V_{\theta} \cos \theta\right)+2 V_{\varphi}\left(\frac{\partial V_{n}}{\partial \varphi}-V_{\varphi} \sin \theta\right)\right]
\end{gathered}
$$

Особый интерес представляют точки (или линии), где кривизна изоэнергетической поверхности обращается в нуль. В этих точках коэффициент усиления имеет сингулярность, указывающую на явления каустики магнонов. Каустика - это особые линии (в двухмерном случае) и особые поверхности, вблизи которых резко возрастает интенсивность спин-волнового поля [15,27]. Кривизна регулярной поверхности (дважды непрерывно дифференцируемой) обращается в нуль в двух случаях [26].

1) В параболических точках. Это такие точки на поверхности, в которых соприкасающийся параболоид вырождается в параболический цилиндр.

2) В точках уплощения. Это такие точки на поверхности, в которых соприкасающийся параболоид вырождается в плоскость (касательную к поверхности). Из этого условия следует, что для любого направления $\mathbf{q}$ коэффициенты $L=P=N=0$.

Нас интересует первый случай. Из выражения для кривизны (26) при заданной частоте спиновых волн $\omega$ получаем условие для нахождения параболических точек, в которых $K_{\omega}(\theta, \varphi)=0$

$$
L N=P^{2}
$$

В случае плоскости грани куба $(\varphi=0)$ или диагональной плоскости $(\varphi=\pi / 4)$ имеют место равенства $V_{\varphi}=\partial V_{n} / \partial \varphi=\partial V_{\theta} / \partial \varphi=0$ и $P=0$, а равенство (29) для нахождения параболических точек преобразуется к виду

$$
V^{2}=V_{\theta} \frac{\partial V_{n}}{\partial \theta}-V_{n} \frac{\partial V_{\theta}}{\partial \theta} .
$$

Поскольку в этих точках кривизна поверхности обращается в нуль, а коэффициент усиления обратно пропорционален $K$, то (29) и (30) - условия для нахождения точек (или линий) каустик, где интенсивность магноного поля резко возрастает. В изотропном случае $(a q \ll 1)$ коэффициент усиления $A=1$. 


\section{4. Фокусировка спиновых волн}

Рассмотрим фокусировку спиновых волн в ферромагнитных кристаллах. В этом случае спектр определяется выражением (18), в котором учитывается только обменное взаимодействие. Величина $\xi$ является варьируемым параметром. Как следует из выражения (19), в длинноволновом приближении спектр магнонов изотропен - он не зависит от направления волнового вектора. Поэтому в случае $q a \ll 1$ фокусировка спиновых волн отсутствует. Зависимость частоты магнонов от направления q возникает во второй половине зоны Бриллюэна $(a q \gtrsim \pi / 2)$.

Определить направления фокусировки и дефокусировки магнонов можно по структуре изоэнергетических поверхностей, поскольку групповая скорость перпендикулярна изоэнергетической поверхности в заданной точке и определяет поток магнонов. Анализ структуры этих поверхностей показал, что в модели, учитывающей взаимодействие только с первыми соседями $(\xi=0)$, в плоскости грани куба $\{100\}(\varphi=0)$ фокусировка магнонов происходит в направлении [110] в области частот $v \geq 3.11 v_{E}$ (см. рис. $\left.1, a\right)$. При $v=4.06 v_{E}$ в окрестности направления [110] изоэнергетическая поверхность представляет собой плоскую поверхность. Поэтому в окрестности направления [110] групповые скорости будут коллинеарны этому направлению. В диагональной плоскости [110] $(\varphi=\pi / 4)$ фокусировка магнонов происходит в том же диапазоне частот в направлении [111] (см. рис. 1,b).

В модели, учитывающей взаимодействие с первыми и следующими за ними соседями с параметром $\xi=0.2$, спектр спиновых волн имеет слабую анизотропию и, соотвественно, слабую фокусировку (см. рис. 2). Этот случай не представляет интереса. Однако в случае $\xi=-0.2$ фокусировка магнонов есть (см. рис. 3). Так же как и в случае $\xi=0$ спиновые волны фокусируются в направлениях [110] и [111], но в области частот $v \geq 0.27 v_{E}$. Причем в этом случае для частот $v \geq 0.53 v_{E}$ на изоэнергетических поверхностях появляются вогнутые области в окрестности направлений фокусировки [110] и [111]. Как мы увидим далее, это приведет к каустике магнонов.

По аналогии с анализом фокусировки фононов [19,20], для магнонов введем углы $\theta_{0}, \theta_{1}, \theta_{2}$ и $\theta_{V 0}$, характеризующие фокусировку спиновых волн при наличии вогнутых участков на изоэнергетической поверхности (см. рис. 4). Эти углы будем отсчитывать от направления фокусировки (в данном случае от направления [110]). Угол $\theta_{1}$ определяет направление волнового вектора, для которого вектор групповой скорости $\mathbf{V}$ параллелен направлению фокусировки. Углы $\theta_{V 0}$ определяют направления групповых скоростей в точках нулевой кривизны на изоэнергетической поверхности. В этих точках вектор групповой скорости магнона имеет максимальное схождение к направлению [110], и этот угол мы обозначим $\theta_{V 0}=\pi / 4-\theta_{V}\left(\theta_{0}\right)$. УГлы $\pm \theta_{0}$ задают направления волновых векторов, соот- ветствующие точкам нулевой кривизны (см. рис. 4). Очевидно, что величину сектора волновых векторов, соответствующих области фокусировки, будут определять групповые скорости $\mathbf{V}\left( \pm \theta_{2}\right)$, коллинеарные направлениям $\mathbf{V}$ в точках нулевой кривизны, а именно $\pi / 4-\theta_{2} \leq \theta \leq \pi / 4+\theta_{2}$ (см. рис. 4). Для нахождения характерных углов $\theta_{i}$ и $\theta_{V 0}$ определим направление групповой скорости $\theta_{V}(\mathbf{q})$ через угловые координаты волнового вектора $\theta$ и $\varphi$. Из условия $\mathbf{V q}=V_{q} \cos (\alpha(\mathbf{q}))$ определим угол между групповой скоростью и волновым вектором: $\alpha(\mathbf{q})= \pm \arccos \left(V_{n}(\mathbf{q}) / V(\mathbf{q})\right)$. Рассмотрим случаи волновых векторов, лежащих в плоскостях $\{100\}$ и $\{110\}$, для которых углы $\varphi=0$ и $\pi / 4$,и компонента групповой скорости $V_{\varphi}=0$. Тогда $\alpha(\mathbf{q})=\arctan \left(V_{\theta}(\mathbf{q}) / V_{n}(\mathbf{q})\right)$. В системе координат с осями по ребрам куба угол между осью $Z$ и направлением групповой скорости равен

$$
\theta_{V}(\mathbf{q})=\theta+\alpha(\mathbf{q})=\theta+\arctan \left(\frac{V_{\theta}(\mathbf{q})}{V_{n}(\mathbf{q})}\right) .
$$

Из последнего равенства следует, что знак компоненты $V_{\theta}$ определяет, в какую сторону будет отклоняться вектор групповой скорости относительно волнового вектора магнона. Поскольку в изотропном случае направления фазовой и групповой скоростей магнона совпадают с направлением волнового вектора, мы получаем возможность рассматривать модель изотропной среды как систему сравнения при анализе влияния фокусировки магнонов на изменение плотности магнонных состояний в анизотропных кристаллах. На рис. 4, $b$ приведена схема расчета характерных углов $\theta_{i}$ и $\theta_{V 0}$. Угол $\theta_{1}$ определяется из условия $\theta_{V}=\pi / 4$ и $\theta=\pi / 4-\theta_{1}$

$$
\theta_{1}=\arctan \left(\frac{V_{\theta}\left(\omega, \pi / 4-\theta_{1}\right)}{V_{n}\left(\omega, \pi / 4-\theta_{1}\right)}\right) .
$$

Для нахождения углов $\theta_{0}$ и $\theta_{V 0}$ необходимо построить функцию $\theta_{V}(\omega, \theta)$ ) при заданной частоте $v=\omega / 2 \pi$ (см. pис. $4, b)$ и найти ее минимум. Положение минимума $d \theta_{V} /\left.d \theta\right|_{\theta=\pi / 4-\theta_{0}}$ определяет точку нулевой кривизны при величине углоа $\theta_{0}$, а значение функции $\theta_{V}\left(\omega, \theta_{0}\right)$ дает величину угла $\theta_{V 0}$ (см. рис. $4, b$ )

$$
\theta_{V 0}=\theta_{0}-\arctan \left(\frac{V_{\theta}\left(\omega, \pi / 4-\theta_{0}\right)}{V_{n}\left(\omega, \pi / 4-\theta_{0}\right)}\right) .
$$

После этого построим векторы групповых скоростей $\mathbf{V}\left( \pm \theta_{0}\right)$ и определим углы „схождения“ групповых скоростей $\pm \theta_{V 0}$. Угол $2 \theta_{V 0}$ определяет область фокусировки магнонов. Очевидно, что величину сектора волновых векторов, соответствующих фокусировке магнонов, будут определять групповые скорости $\mathbf{V}\left( \pm \theta_{2}\right)$, коллинеарные направлениям $\mathbf{V}$ в точках нулевой кривизны, а именно, $\pi / 4-\theta_{2} \leq \theta \leq \pi / 4+\theta_{2}$ (см. рис. 4). Уравнение для определения угла $\theta_{2}$ имеет вид

$$
\theta_{V 0}=\theta_{2}-\arctan \left(\frac{V_{\theta}\left(\omega, \pi / 4-\theta_{2}\right)}{V_{n}\left(\omega, \pi / 4-\theta_{2}\right)}\right) .
$$



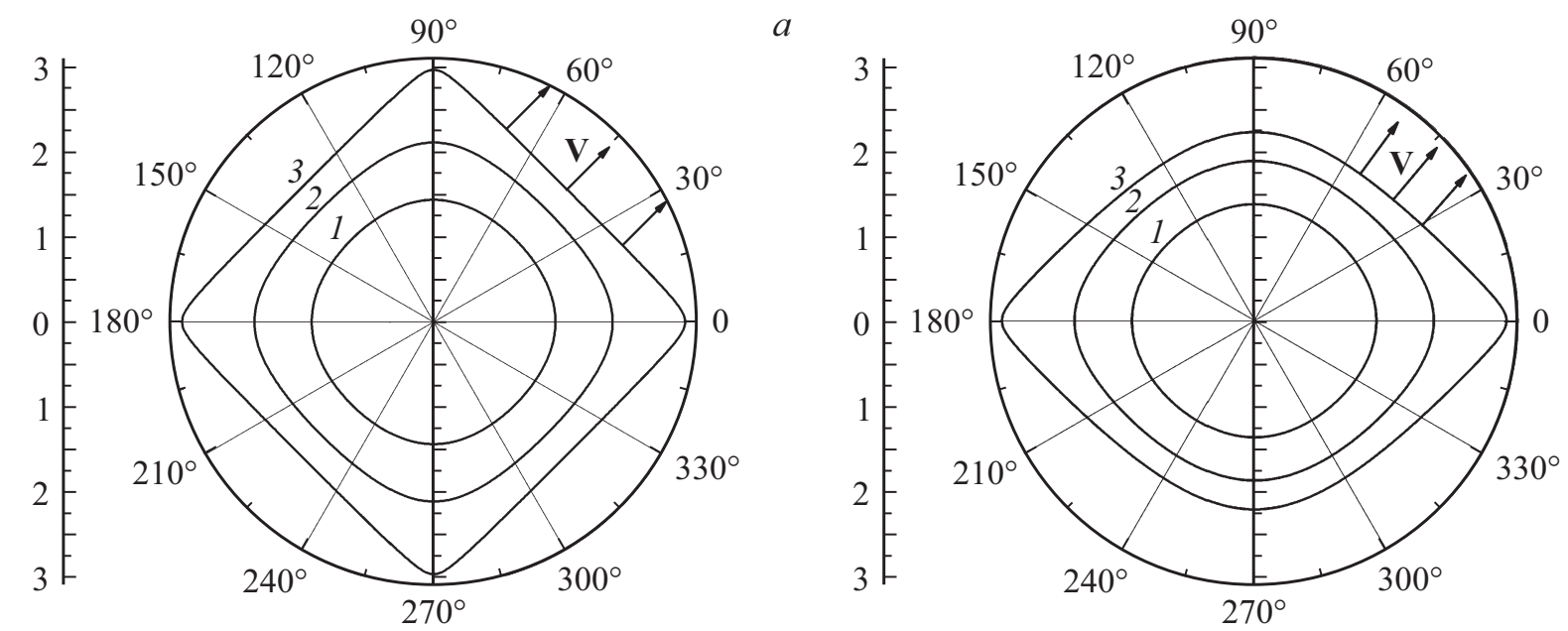

$b$

Рис. 1. Сечения поверхностей постоянной частоты $a q(\theta)$ плоскостями $\{100\}(a)$ и $\{110\}(b)$ в модели, учитывающей только

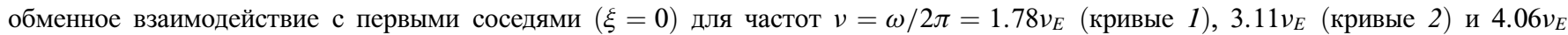
(кривые 3). Стрелками изображены направления групповых скоростей $\mathbf{V}$ в областях фокусировки магнонов.
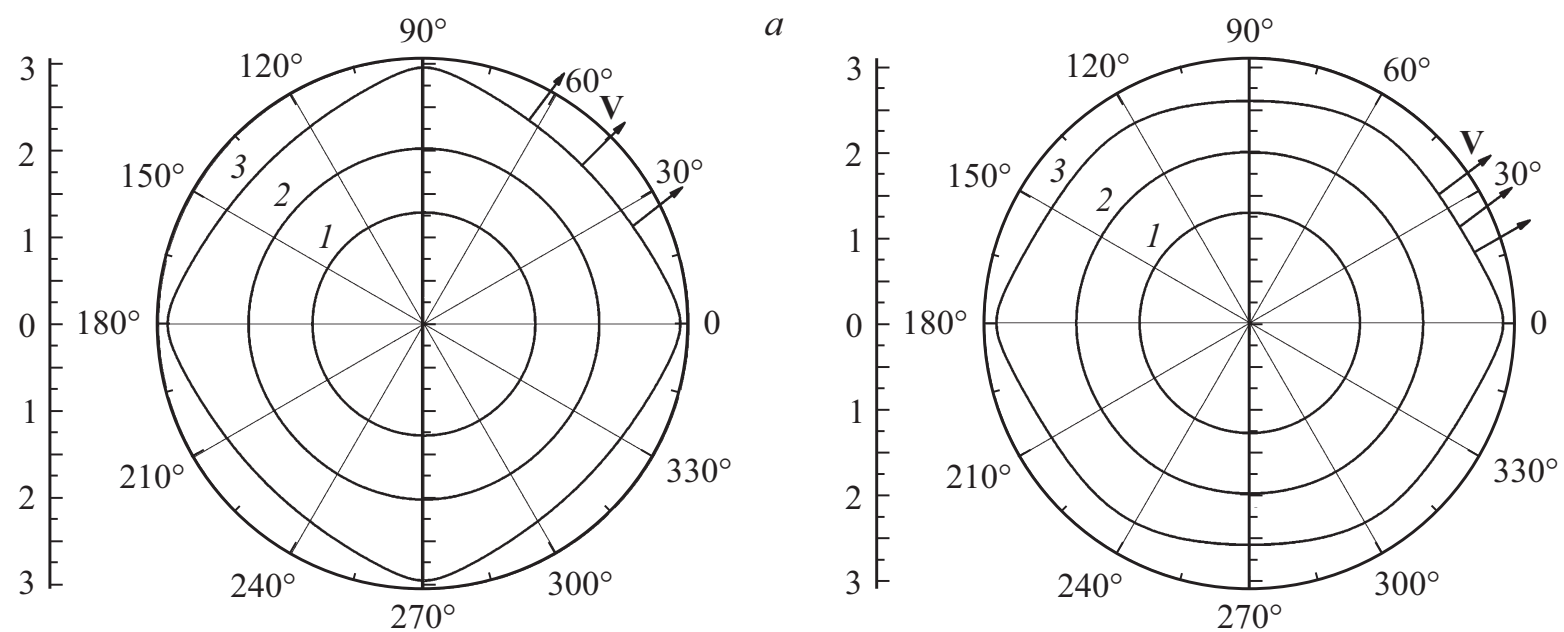

$b$

Рис. 2. Сечения поверхностей постоянной частоты $a q(\theta)$ плоскостями $\{100\}(a)$ и $\{110\}(b)$ в модели, учитывающей обменное взаимодействие с первыми и вторыми соседями с параметром $\xi=0.2$ для частот $v=\omega / 2 \pi=2.78 v_{E}($ кривые 1$), 5.56 v_{E}($ кривые 2$)$ и $7.33 v_{E}$ (кривые 3 ). Стрелками изображены направления групповых скоростей $\mathbf{V}$ в областях фокусировки магнонов.
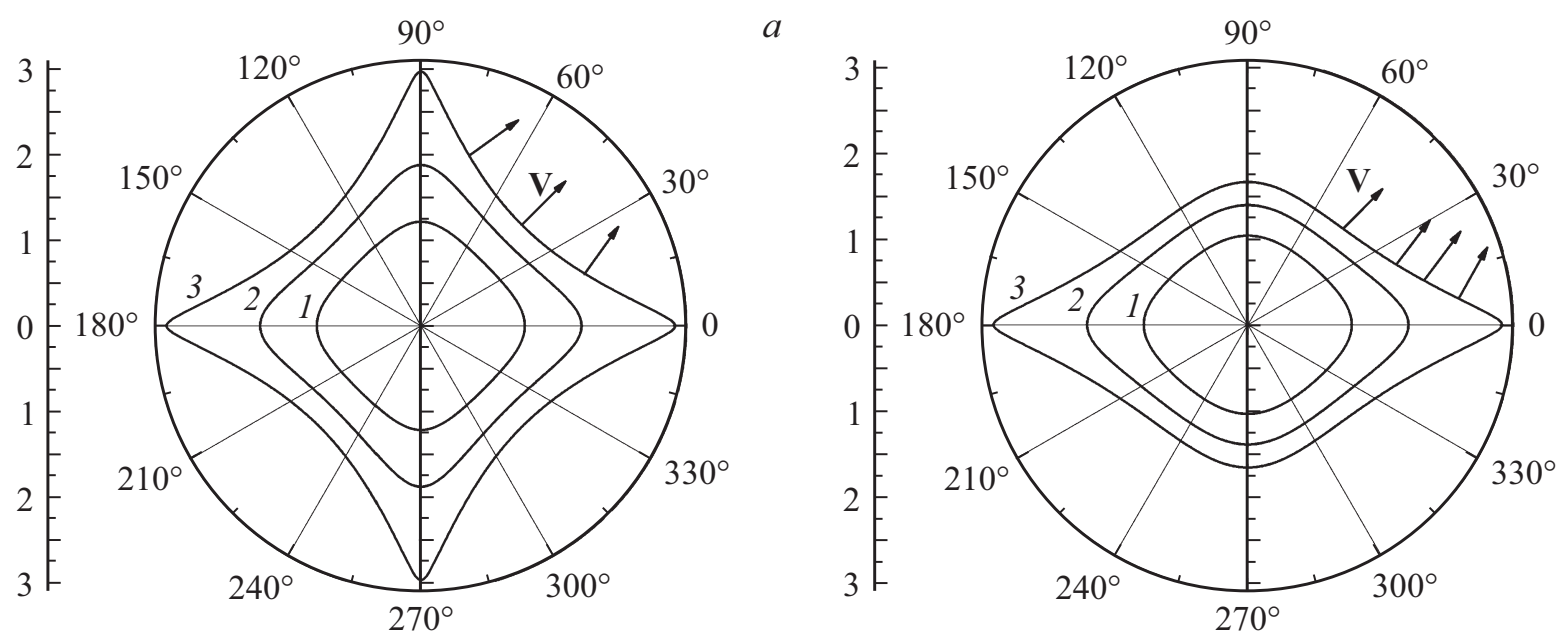

$b$

Рис. 3. Сечения поверхностей постоянной частоты $a q(\theta)$ плоскостями $\{100\} \quad(a)$ и $\{110\}(b)$ в модели, учитывающей обменное взаимодействие с первыми и вторыми соседями с параметром $\xi=-0.2$ для частот $v=\omega / 2 \pi=0.27 v_{E}$ (кривые 1 ), $0.53 v_{E}$ (кривые 2) и $0.83 v_{E}$ (кривые 3 ). Стрелками изображены направления групповых скоростей $\mathbf{V}$ в областях фокусировки магнонов. 

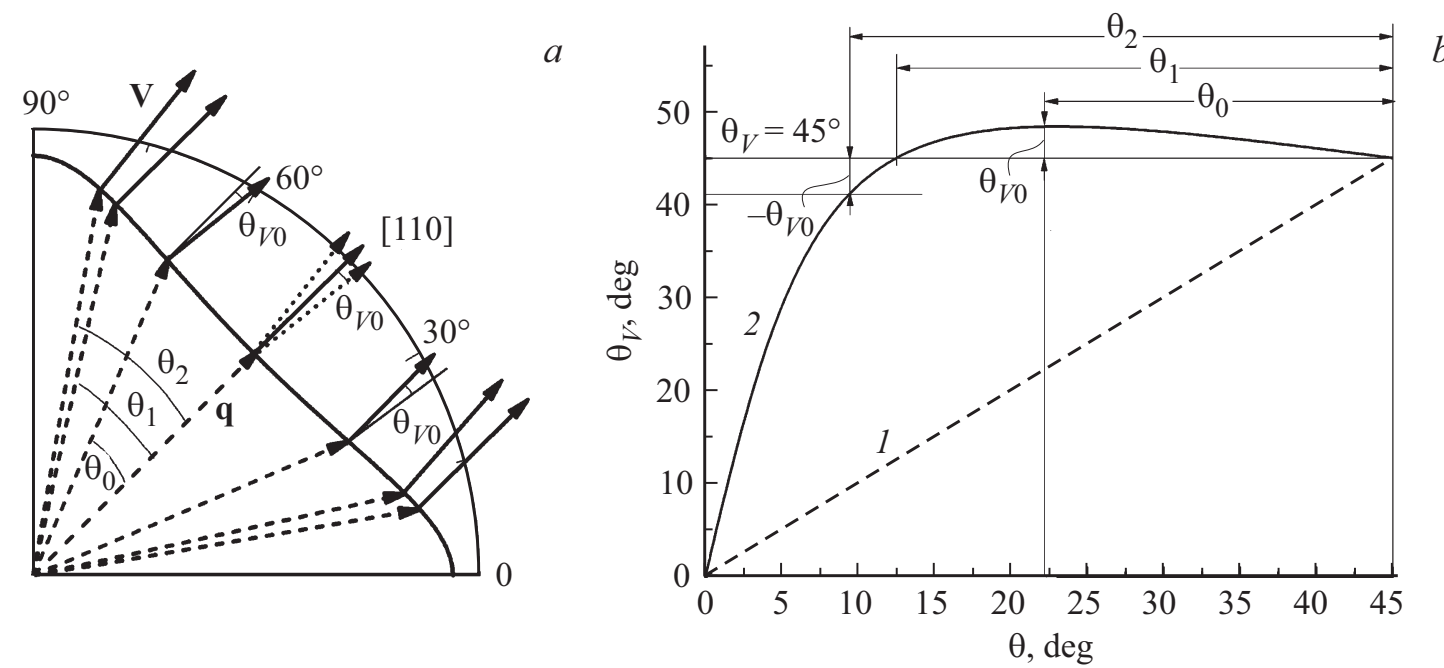

Рис. 4. Схема расчета характерных углов $\theta_{i}$ и $\theta_{V 0}$ в плоскости грани куба для ферромагнетика с параметром $\xi=-0.2$ и частотой спиновых волн $v_{0}=0.53 v_{E}$. Рисунок $(a)-$ сечение изоэнергетической поверхности плоскостью грани куба. Штриховыми стрелками изображены волновые векторы $\mathbf{q}$, сплошными стрелками - соответствующие им групповые скорости $\mathbf{V}$, перпендикулярные поверхности постоянной частоты. Рисунок $(b)-$ зависимости угла $\theta_{V}(\theta)$ : кривая $1-\theta_{V}(\theta)=\theta-$ изотропный случай $(a q \ll 1)$ и кривая $2-$ для частоты $v=0.53 v_{E}$.

Для графического определения угла $\theta_{2}$ на рисунке $4, b$ проводим горизонтальную линию на высоте $\pi / 4-\theta_{V 0}$ до пересечения с кривой 2 (см. pис. $4, b)$. Точка пересечения $\theta_{V}\left(\theta_{2}\right)=\pi / 4-\theta_{V 0}$ даст нам угол $\theta_{2}$, который определяет сектор фокусировки $\pi / 4-\theta_{2} \leq \theta \leq \pi / 4+\theta_{2}$ в пространстве волновых векторов. Таким образом, расходящийся сектор волновых векторов $\pi / 4-\theta_{2} \leq \theta \leq \pi / 4+\theta_{2}$ в плоскости $\{100\}$ превращается в сходящийся к направлению фокусировки [110] сектору групповых скоростей $\pi / 4-\theta_{V 0} \leq \theta_{V} \leq \pi / 4+\theta_{V 0}$.

\section{1. Плотность состояний магнонов}

По аналогии с анализом фокусировки фононов [19,20], для магнонов введем среднюю плотность состояний для волновых векторов в плоскости $\{100\}$, приходящуюся на единичный угол в областях фокусировки $\pi / 4-\theta_{V 0} \leq \theta_{V} \leq \pi / 4+\theta_{V 0}$ и в изотропной среде $\pi / 4-\theta_{2} \leq \theta \leq \pi / 4+\theta_{2}$. Поскольку для изотропной среды расходящийся сектор волновых векторов в плоскости $\{100\}$ с углом $2 \theta$ (см. табл. 1) превращается

Таблица 1. Характерные углы $\theta_{i}, \theta_{V 0}$ и плотность состояний магнонов $n_{F I}$ в направлении фокусировки [110] и $N_{D I}$ в направлении дефокусировки [100] в плоскости грани куба для ферромагнетика с параметром $\xi=-0.2$ и частотой спиновых волн $v_{0}=0.53 v_{E}$ и $0.83 v_{E}$

\begin{tabular}{c|c|c|c|c|c|c|c}
\hline$v_{0} / v_{E}$ & $\theta_{0}$ & $\theta_{1}$ & $\theta_{2}$ & $\theta_{V 0}$ & $n_{F I}$ & $n_{D I}$ & $n_{F D}$ \\
\hline 0.53 & $21.9^{\circ}$ & $32.6^{\circ}$ & $35.3^{\circ}$ & $3.4^{\circ}$ & 10.4 & 0.23 & 45.4 \\
0.83 & $39.7^{\circ}$ & $44.2^{\circ}$ & $44.6^{\circ}$ & $18.4^{\circ}$ & 2.43 & 0.014 & 174
\end{tabular}

в расходящийся сектор векторов групповых скоростей фононов с углом $2 \theta_{V 0}$, то средняя плотность состояний в областях фокусировки магнонов будет больше, чем в изотропной среде в отношении

$$
n_{F I}=\frac{N_{F}}{N_{\text {Iso }}}=\frac{\theta_{2}}{\theta_{V 0}} .
$$

Для области дефокусировки средняя плотность состояний $n_{D 1}$ будет меньше, чем $N_{\text {Iso }}$ в отношении

$$
n_{D I}=\frac{N_{D}}{N_{\text {Iso }}}=\frac{\pi-4 \theta_{2}}{\pi-4 \theta_{V 0}} .
$$

Из формул (35) и (36) получим отношения средних плотностей состояний для областей фокусировки и дефокусировки магнонов, которые характеризуют анизотропию плотности состояний:

$$
n_{F D}=\frac{N_{F}}{N_{D}}=\frac{\theta_{2}\left(\pi-4 \theta_{V 0}\right)}{\theta_{V 0}\left(\pi-4 \theta_{2}\right)}
$$

Предложенный метод анализа фокусировки спиновых волн позволил определить угловые распределения средних плотностей магнонных состояний для областей фокусировки и дефокусировки магнонов и оценить величины этих плотностей в плоскости грани куба для двух частот спиновых волн $v=0.53 v_{E}$ и $0.83 v_{E}$. (см. табл. 1 ). Поскольку для частоты $v=0.53 v_{E}$ изоэнергетическая поверхность в окрестности направления [110] имеет более плоский участок, чем для частоты $v=0.83 v_{E}$, то и плотность магнонных состояний в первом случае в 4.3 раза больше, чем во втором.

Заметим, что этот метод разработан для конкретного сечения изоэнергетической поверхности $\varphi=$ const, поэтому компонента групповой скорости $V_{\varphi}$ и ее производные не учитываются. Кроме того он применим только 

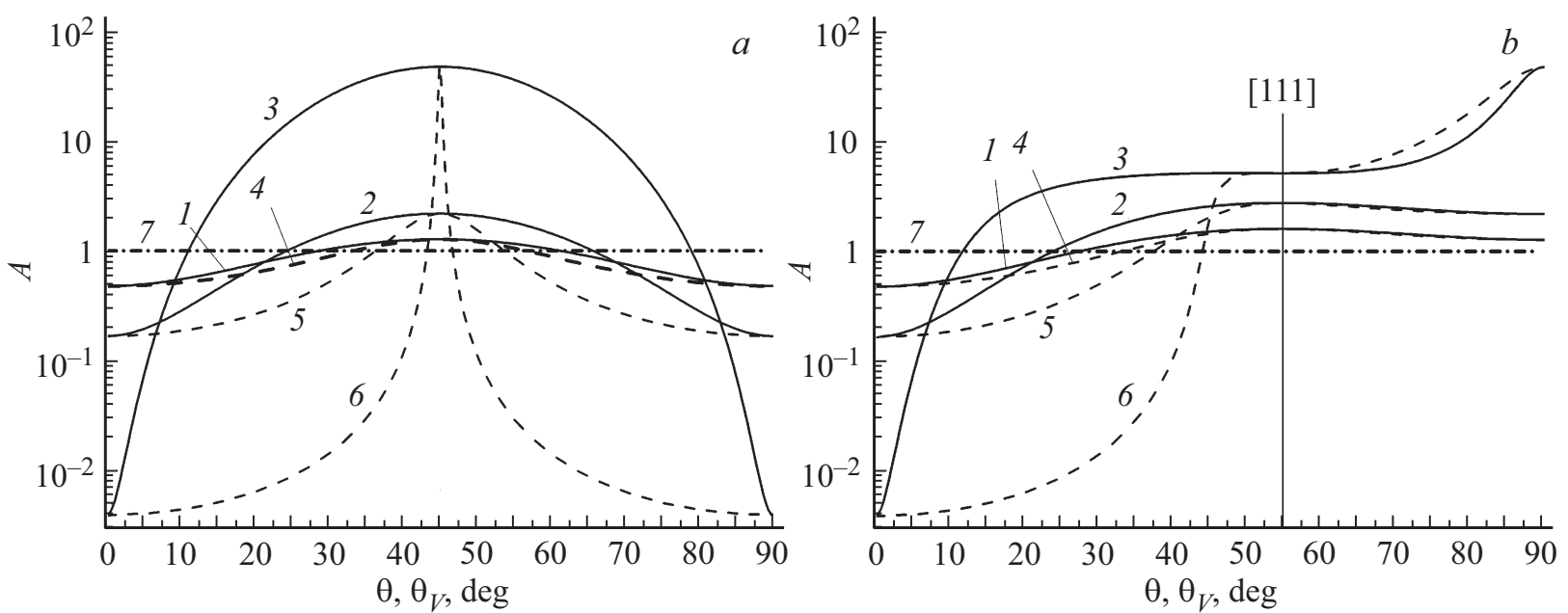

Рис. 5. Угловые зависимости коэффициента усиления $A(\theta)$ (кривые $1,2,3)$ и $A\left(\theta_{V}\right)$ кривые $\left.4,5,6\right)$ в плоскости грани куба $(a)$ и в диагональной плоскости $(b)$ для параметра $\xi=0$ (учет обменного взаимодействия с ближайшими соседями) для частот спиновых

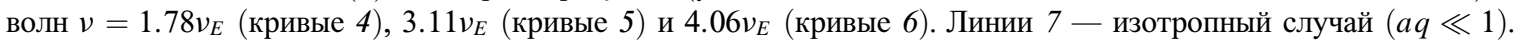
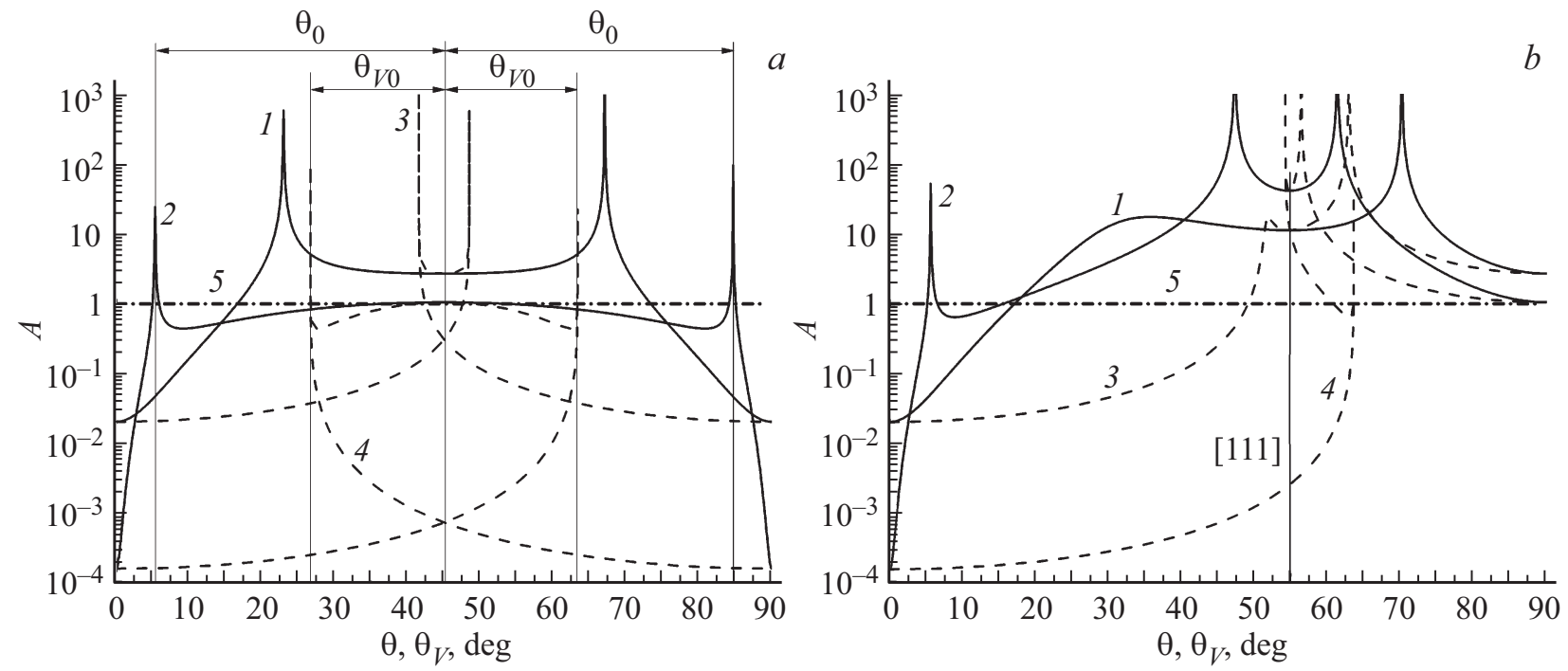

Рис. 6. Угловые зависимости коэффициента усиления $A(\theta)$ (кривые 1,2$)$ и $A\left(\theta_{V}\right)$ (кривые 3,4$)$ в плоскости грани куба $(a)$ и в диагональной плоскости $(b)$ для параметра $\xi=-0.2$ для частот спиновых волн $v_{0}=0.53 v_{E}($ кривые 1,3$)$ и $v=0.83 v_{E}$ (кривые 2,4$)$. Линии 5 - изотропный случай $(a q \ll 1)$. Углы $\theta_{0}$ и $\theta_{V 0}$ соответствуют особым точкам коэффициента усиления.

в случаях, когда на изоэнергетической поверхности имеются вогнутые участки.

\section{2. Коэффициент усиления спиновых волн}

В разделе 3 введен коэффициент усиления $A$ спиновых волн и приведены полученные для него аналитические выражения (25)-(28). В этом разделе приведем результаты расчета $A$ для ферромагнетика со спектром (18) в плоскости грани куба и в диагональной плоскости для значений параметра обменного взаимодействия со вторыми соседями $\xi=0,0.2$ и -0.2 . Для этих случаев в табл. 2 приведены значения коэффициента усиления в симметричных направлениях. Наибольшее значение величина $A=47.5$ имеет в направлении фокусиров- ки [110], а наименьшее $-A=0.004$ в направлении дефокусировки [100] для параметра $\xi=0$ при частоте спиновых волн $v_{0}=4.06 v_{E}$. В этом случае интенсивность потока магнонов при переходе от направления [100] к направлению [110] возрастает в $10^{4}$ раз. Как отмечалось ранее, для параметра $\xi>0$ спектр магнонов имеет меньшую анизотропию и, соответственно, более слабую фокусировку (см. табл. 2).

Непосредственное вычисление коэффициента усиления по формулам $(25)-(28)$ дает значения $A$ в пространстве волновых векторов (q-пространстве). Однако для экспериментальных приложений необходимо знать эту величину в заданном направлении в координатном пространстве (V-пространстве). Поэтому на рис. 5 и 6 представлены угловые зависимости коэффициента уси- 
Таблица 2. Коэффициенты усиления магнонов в симметричных направлениях для различных частот спиновых волн $v / v_{E}$ и параметров обменного взаимодействия со вторыми соседями $\xi$

\begin{tabular}{c|c|c|r|r}
\hline$\xi$ & $v / v_{E}$ & $A_{[100]}$ & $A_{[110]}$ & $A_{[11]}$ \\
\hline \multirow{3}{*}{0} & 1.78 & 0.48 & 1.27 & 1.60 \\
& 3.11 & 0.17 & 2.19 & 2.76 \\
& 4.06 & 0.004 & 47.52 & 5.13 \\
\hline \multirow{3}{*}{0.2} & 2.78 & 0.78 & 1.09 & 1.15 \\
& 5.56 & 0.38 & 1.47 & 1.43 \\
& 7.33 & 0.01 & 3.29 & 1.83 \\
\hline & 0.27 & 0.11 & 4.12 & 3.95 \\
-0.2 & 0.53 & 0.02 & 2.70 & 11.27 \\
& 0.83 & $1.6 \cdot 10^{-4}$ & 1.052 & 41.48
\end{tabular}

ления для обоих случаев: $A(\theta)$ (сплошные кривые) и $A\left(\theta_{V}\right)$ (штриховые кривые).

\section{3. Каустика магнонов}

Случай отрицательного параметра $\xi$ представляет особый интерес, поскольку в параболических точках (точки, в которых кривизна изоэнергетической поверхности обращается в нуль) коэффициент усиления имеет сингулярность $(A \rightarrow \infty)$. Эти точки указывают на каустику магнонов.

Как видно из рис. 6, $a$ в плоскости грани куба при $\theta=\pi / 4-\theta_{0}$ коэффициент усиления $A \rightarrow \infty$ (сплошные кривые 1 и 2). Этому углу соответствует направление групповой скорости с углом $\theta_{V}=\pi / 4-\theta_{V 0}$ (штриховые кривые 3 , и 4). В этом направлении в плоскости грани куба будет наблюдаться интенсивный рост потока магнонов (или каустика). Углы $\theta_{0}$ и $\theta_{V 0}$ приведены в табл. 1.

В диагональной плоскости для параметра $\xi=-0.2$ и частоты спиновой волны $v=0.53 v_{E}$ наблюдается один пик интенсивности спиновой волны между направлениями [110] и [111], соответствующий каустике магнонов. Однако с увеличением частоты спиновой волны появляются еще два пика. Для частоты спиновой волны $v=0.83 v_{E}$ в $\mathbf{q}$-пространстве один пик интенсивности имеет место вблизи направления [100], а два других - по обе стороны от направлени [111]. Однако в $\mathbf{V}$-пространстве все три пика находятся в окрестности направления [111] (см. рис. 6, b).

В качестве примера рассмотрим одноосный ферромагнитный кристалл ЖИГ, который используется в наноспинтронике. Для него известны параметры, определяющие спектр магнонов (18): $v_{E}=\omega / 2 \pi=0.18 \mathrm{THz}$, $a=12.376 \AA$ [28]. В этом кристалле фокусировка спиновых волн будет наблюдаться на частотах порядка $0.048-1.3 \mathrm{THz}$. В случае обменного взаимодействия магнитного момента только с первыми соседями $(\xi=0)$ плоский участок на изоэнергетической поверхности и, соответственно сильная фокусировка, будут иметь место на частоте $v=4.06 v_{E}=0.73 \mathrm{THz}$. Обменное взаимодействие с первыми и вторыми соседями с параметром $\xi=0.2$ приводит к фокусировке и формированию каустики магнонов на частотах $v \gtrsim 0.095 \mathrm{THz}$.

\section{5. Выводы}

В настоящей работе мы проанализировали процесс передачи энергии из точечного источника спиновых волн в объемном кристалле и показали, что в длинноволновом приближении фокусировка обменных спиновых волн отсутствует: она наблюдается лишь для волновых векторов во второй половине зоны Бриллюэна. Для ее реализации в магнитно-одноосных кристаллах не требуется внешнего магнитного поля и магнитостатических эффектов.

Основные результаты работы можно сформулировать следующим образом:

1. Эффект фокусировки спиновых волн зависит от знака и величины параметра обменного взаимодействия магнитного момента со вторыми соседями $\xi$. В частности, для $\xi<0$ в окрестности направлений [110] и [111] (в точках нулевой кривизны поверхности постоянной энергии) имеет место каустика магнонов. Вблизи этих точек (или линий) резко возрастает интенсивность потока спиновых волн.

2. Показано, что в простой кубической решетке в магнитно-одноосном магнетике с обменным взаимодействием магнитного момента с ближайшими и следующими за ними соседями магноны фокусируются вдоль направлений [110] и [111], а в направлениях [100] дефокусируются.

3. Также обнаружено, что ни внешнее магнитное поле, ни поле магнитной анизотропии не приводят к изменению направлений фокусировки магнонов.

\section{Список литературы}

[1] B. Taylor, H.J. Maris, C. Elbaum. Phys. Rev. Lett. 23, 416 (1969).

[2] B. Taylor, H.J. Maris, C. Elbaum. Phys. Rev. B 3, 1462 (1971).

[3] J.P. Wolfe. Imaging Phonons. Acoustic Wave Propagation in Solids. Cambridge University Press, N.Y. (1998). 411 p.

[4] H.J. Maris. J. Acoust. Soc. Am. 50, 812 (1971).

[5] Cz. Jasiukiewicz, T. Paszkiewicz, D. Lehmann. Z. Phys. B Condens. Matter 96, 2, 213 (1994).

[6] E. Held, W. Klein, R.P. Huebener. Z. Phys. B Condens. Matter 75, 2, 223 (1989).

[7] O.Büttner, M. Bauer, S.O. Demokritov, B. Hillebrands, Yu.S. Kivshar, V. Grimalsky, Yu. Rapoport, M.P. Kostylev, B.A. Kalinikos, A.N. Slavin. J. Appl. Phys. 87, 9, 5088 (2000).

[8] V.E. Demidov, S.O. Demokritov, K. Rott, P. Krzysteczko, G. Reiss. Appl. Phys. Lett. 91, 252504 (2007).

[9] V. Veerakumar, R.E. Camley. Phys. Rev. B 74, 214401 (2006).

[10] V. Veerakumar, R.E. Camley. Phys. Rev. B 81, 174432 (2010).

[11] J.J. Bible, R.E. Camley. Phys. Rev. B 95, 22, 224412 (2017). 
[12] C.S. Davies, A.V. Sadovnikov, S.V. Grishin, Yu.P. Sharaevskii, S.A. Nikitov, V.V. Kruglyak. Appl. Phys. Lett. 107, 16, 162401 (2015).

[13] R. Gieniusz, H. Ulrichs, V.D. Bessonov, U. Guzowska, A.I. Stognii, A. Maziewski. Appl. Phys. Lett. 102, 10, 102409 (2013).

[14] Е.А. Туров. Физические свойства магнитоупорядоченных кристаллов. Изд-во АН СССР, М. (1963). 223 с.

[15] A.G. Every. Phys. Rev. B 24, 3456 (1981).

[16] J. Philip, K.S. Viswanathan. Phys. Rev. B 17, 12, 4969 (1978).

[17] M. Lax, V. Narayanamurti. Phys. Rev. B 22, 4876 (1980).

[18] G.A. Northrop, J.P. Wolfe. Phys. Rev. B 22, 6196 (1980).

[19] I.I. Kuleyev, S.M. Bakharev, I.G. Kuleyev, V.V. Ustinov. Phys. Status Solidi 59, 1600263 (2017).

[20] И.И. Кулеев, С.М. Бахарев, И.Г. Кулеев, В.В. Устинов. Физика металлов и металловедение 118, 1, 12 (2017).

[21] Я. Смит, Х. Вейн. Ферриты. ИЛЛ, М. (1962). 504 с.

[22] F.H. de Leeuw, R. Van den Doel, V. Enz. Rep. Progr. Phys. 43, 691 (1980).

[23] Т. О’Делл. Ферромагнитодинамика. Динамика ЦМД, доменов и доменных стенок. Мир, М. (1983). 253 с.

[24] Ч. Киттель. Квантовая теория твердых тел. Наука, М. (1967). 492 с.

[25] Ферромагнитный резонанс. Сборник статей / Под ред. С.В. Вонсовского. ГИФМЛ, М. (1961). 343 с.

[26] А.В. Погорелов. Дифференциальная геометрия. Наука, М. (1974). $176 \mathrm{c}$.

[27] В.И. Арнольд. Теория катастроф. Наука, М. (1990). 128 с.

[28] V. Cherepanov, I. Kolokolov, V. L'vov. Phys. Rep. 229, 81 (1993).

Редактор Е.Ю. Флегонтова 